\title{
Author Correction: High carrier mobility in graphene doped using a monolayer of tungsten oxyselenide
}

Min Sup Choi, Ankur Nipane, Brian S. Y. Kim, Mark E. Ziffer, Ipshita Datta, Abhinandan Borah, Younghun Jung, Bumho Kim, Daniel Rhodes, Apoorv Jindal, Zachary A. Lamport, Myeongjin Lee, Amirali ZangiabadiD, Maya N. Nair (D, Takashi Taniguchi (D), Kenji Watanabe (D), Ioannis Kymissis, Abhay N. Pasupathy (D), Michal Lipson (D), Xiaoyang Zhu, Won Jong Yoo iD, James Hone (D) and James T. Teherani (D)

Correction to: Nature Electronics https://doi.org/10.1038/s41928-021-00657-y, published online 22 October 2021.

In the version of this article initially published, the first grant listed in the following was missing the final digit: "This work was primarily supported by the NSF MRSEC Program at Columbia in the Center for Precision-Assembled Quantum Materials (DMR-2011736 and DMR-1420634)." The error has been corrected in the online version of the article.

Published online: 24 November 2021

https://doi.org/10.1038/s41928-021-00696-5

(๑) The Author(s), under exclusive licence to Springer Nature Limited 2021 